\title{
SCALE-DEPENDENCE OF BIAS FROM APM-BGC SAMPLE
}

L. Z. FANG

Dept. of Physics, University of Arizona, Tucson, USA

Z. G. DENG

Dept. of Physics, Graduate School, USTC, P. R. China

AND

X. Y. XIA

Dept. of Physics, Tianjin Normal University, P.R.China

Applying a discrete wavelet transformation (DWT) ${ }^{1}$ to the spiral (SP) and elliptical+lenticular (EL) galaxies in APM bright galaxy catalog (APMBGC $)^{2}$, we investigated the scale-dependence of bias parameter $b$ in the linear bias model $(\delta \rho(\mathbf{x}) / \bar{\rho})_{g}=b(\delta \rho(\mathbf{x}) / \bar{\rho})_{m}$.

We showed that the scale-dependence of bias cannot simply be described by one parameter $b$. The linear bias model actually assumed that all $j$ spectra of the DWT-coefficient-represented bias parameters $\tilde{b}_{j}^{(n)}$ and $b_{j}^{(n)}$, where $n$ and $j$ are integers, are flat. Since these parameters are statistically independent, one cannot draw a general conclusion on either bias scaleindependence or dependence from one or a few statistics. A systematical detection of the $j$ spectrum is necessary.

The $j$-spectrum analysis of the APM-BGC galaxies shows that the scaleindependent bias model is consistent with the SP and EL distributions if only statistics of 2-point correlation function or Fourier power spectrum are involved. The bias scale-dependence becomes, however, substantial when phase-sensitive statistics are applied. This result indicates that the bias scale-dependence is essentially related to the non-Gaussianity of galaxy distribution, i.e. the non-linear and non-local relationship between galaxy formation and their environment.

\section{References}

1. Fang, L.Z. \& Pando, J. 1997, The 5th Current Topics of Astrofundamental Physics, eds. N.Sanchez \& A.Zichichi, World Scientific; 1996, ApJ, 459, 1

2. Loveday, L. (1996) MNRAS, 278, 1025 